Similarly fish oil supplementation and probiotics may also decrease risk of polyp development. Daily supplementation with multivitamins may increase polyp risk, although this is complex and multi-factorial. Large scale extension of this study needs to be undertaken to consolidate the pilot study findings.

Competing interests None.

Keywords colonic polyps, colorectal cancer, vitamin supplementation.

\section{PTU-068 THE EFFECT OF DIETARY FACTORS AND VITAMIN SUPPLEMENTATION ON POLYP SIZE, FREQUENCY, AND DYSPLASIA IN A REGIONAL COLORECTAL CANCER SCREENING COHORT}

doi:10.1136/gut.2011.239301.196

N E Burch, ${ }^{1,2^{*}} \mathrm{~J}$ Huber $^{2}{ }^{1}$ Digestive Diseases Department, University Hospitals Leicester NHS Trust, Leicester, UK; ${ }^{2}$ Health Sciences Research Centre, Roehampton University, London, UK

Introduction This pilot study aimed to evaluate the feasibility of detailed dietary evaluation in patients who have undergone colonoscopy as part of the National Bowel Cancer Screening Program (BCSP). This dietary data was then correlated with colonoscopy outcomes to identify any potential dietary factors which may impact on risk of polyp development.

Methods 150 patients were posted a recruitment pack containing a 3-day food diary and vitamin supplementation questionnaire. Colonoscopy results for each patient were summarised with respect to polyp incidence, polyp characteristics, and other diagnoses. Completed food diaries were analysed to establish average daily macro and micro-nutrient composition using 'Dietplan 6.0' software. Additional dietary supplement use was summarised from questionnaire responses to evaluate nature, frequency, and duration of supplement use. All dietary data were then evaluated with respect to colonoscopy outcomes to establish any potential associations. The control group was defined as those without polyps at their screening colonoscopy.

Results A total of $30 \%$ of the research cohort returned a completed postal food diary and vitamin questionnaire. Individuals with polyps were more likely to participate (OR 1.56). A significant proportion regularly ingested dietary supplements such as multivitamins (29\%), fish oils (24\%), and probiotics $(27 \%)$. There was a non-significant trend towards increased polyp incidence in those taking vitamin supplementation (OR 1.41). In contrast there was an apparent decreased polyp incidence in those taking fish oil supplements (0.76), and probiotics (OR 0.69). Higher vitamin $\mathrm{E}$ intake appeared to be protective against polyp development, with the effect approaching significance following independent samples t test $(p=0.09)$. Increased average calcium intake was also associated with a non-significant trend towards decreased polyp incidence $(p=0.17)$. Both cholesterol and fat intake were associated with a non-significant increased risk of polyps $(p=0.15$, and 0.16 respectively).

Conclusion The BCSP population provides a unique platform for evaluation of dietary factors associated with colonic polyp development. This pilot study demonstrates an effective method for evaluation of these dietary factors, and despite relatively small numbers has shown some important trends which approach significance. Higher dietary intakes of vitamin $\mathrm{E}$ and calcium may protect against polyp development. 